\title{
Development of Genetic Stocks and Biochemical Markers to Facilitate Utilization of Aegilops variabilis in Wheat Improvement
}

\author{
M. D. H. M. William and A. Mujeeb-Kazi* \\ International Maize and Wheat Improvement Center (CIMMYT), Lisboa 27, \\ Apartado Postal 6-641 06600 México, D.F., Mexico
}

Accepted September 6, 1995

Aegilops variabilis $(2 \mathrm{n}=4 \mathrm{x}=28$, UUSS $)$ accession $13 \mathrm{E}$ is resistant to Karnal bunt (Warham et al. 1986). With the objective of transferring this attribute to wheat (Triticum aestivum L.; AABBDD, $2 \mathrm{n}=6 \mathrm{x}=42$ ) we have a dual program underway to (i) produce disomic chromosome additions of Ae. variabilis in T. aestivum and (ii) manipulate homoeologous group introgressions from Ae. variabilis to wheat through the recessive $p h$ locus of $T$. aestivum cv. Chinese Spring initiated at the $F_{1}$ hybrid stage, advanced by backcrosses to elite wheat cultivars and ending with genetic stability. For both situations to materialize, it is necessary to have a wide range of diagnostic markers for detecting alien chromosomal presence, establishing homoeology and eventually tracking cryptic alien genetic introgressions. Though cytological and/or morphological parameters have been used to establish homoeology (MujeebKazi and Asiedu 1990) a more effective way is by utilizing biochemical markers; of which proteins and isozymes are an option. We have conducted these biochemical analyses on the amphiploids of $T$. aestivum/Ae. variabilis and T. turgidum $\mid$ Ae. variabilis through the application of seven markers; high molecular weight glutenin (HMW-Glu), glucose phosphate isomerase (GPI), seed esterase (EST-5), 6-phosphogluconate dehydrogenase (6-PGD), $\beta$-amylase ( $\beta$-AMY), glutamate oxaloacetate transaminase (GOT) and $\alpha$-amylase ( $\alpha$-AMY). These results form the basis of this paper.

\section{Materials and methods}

Germplasm. Seeds of Aegilops variabilis ( $2 \mathrm{n}=4 \mathrm{x}=28$, UUSS), CIMMYT Wide Crosses accession 13E, were the source for biochemical analyses and hybridization following the prior determination of its Karnal bunt resistance status (Warham et al. 1986). A composite of 5 seeds were analyzed for HMW-glutenins, GPI, EST-5, 6-PGD, $\beta$-AMY and $\alpha$-AMY. Leaf extracts from young seedlings were analyzed for GOT. Triticum aestivum cvs. Chinese Spring (CS), Alondra/Pavon "S", T. turgidum cv Laru "S", amphiploids of the T. aestivum and T. turgidum cultivars with Ae. variabilis $(2 \mathrm{n}=10 \mathrm{x}=70$ and $2 \mathrm{n}=8 \mathrm{x}=56$, AABBDDUUSS and AABBUUSS) were additionally utilized in the study. The amphiploids would determine whether the biochemical markers specific to Ae. variabilis were expressed in the wheat backgrounds.

Enzyme extraction. Mature seeds were used for all systems except for GOT and $\alpha$-AMY that utilized young leaves from rapidly growing seedlings (GOT) or 6 day old germinating seeds ( $\alpha$-AMY) respectively. Individual bread wheat, durum wheat and Ae. variabilis seeds were ground and extracted with $200 \mu 1$ of $0.01 \mathrm{M}$ Tris- $\mathrm{HCl}(\mathrm{pH} \mathrm{7.5)}$ for GPI and 6-PGD. The extraction buffer for $\beta$-AMY was $0.01 \mathrm{M}$ Tris- $\mathrm{HCl}(\mathrm{pH} 7.5)$ with $0.2 \mathrm{M} 2$-mercaptoethanol and

* Corresponding author: A. Mujeeb-Kazi. 
$10 \%$ sucrose, of which $500 \mu 1$ was utilized to extract the mature kernel. GPI and EST-5 extractions were done at $4^{\circ} \mathrm{C}$ for $1 \mathrm{hr}$, while those for 6-PGD and $\beta$-AMY were overnight at $4^{\circ} \mathrm{C}$. For $\alpha$-AMY the 6 day old germinating seeds were extracted with $0.5 \mathrm{M}$ Tris- $\mathrm{HCl}(\mathrm{pH}$ $7.5)+0.002 \mathrm{M} \mathrm{CaCl}_{2}$. The extracts were heated for $10 \mathrm{~min}$ at $70^{\circ} \mathrm{C}$ to inactivate $\beta$-AMY. Leaves from young, fast growing seedlings were macerated with sand by a mortar and pestle, extracted with $0.05 \mathrm{M}$ Tris- $\mathrm{HCl}(\mathrm{pH} 7.5)$ containing $10 \%$ sucrose and immediately used for GOT analysis. All samples were centrifuged for $2 \mathrm{~min}$ at room temperature prior to electrophoresis.

Isoelectric focusing. Polyacrylamide gel isoelectric focusing (IEF) was used for EST-5, GPI, $\alpha$-AMY and $\beta$-AMY. EST-5 and GPI isozyme separations were on $\mathrm{pH} 3.5-9.5$ gels while those of $\alpha$-AMY and $\beta$-AMY were on $\mathrm{pH} 4.0-6.5$ gels. The anode/cathode buffer solutions and the electrophoretic details were similar to those previously described (William and MujeebKazi 1993).

Native polyacrylamide gel electrophoresis. Discontinuous native polyacrylamide gel electrophoresis (native-PAGE) was used for the separation of 6-PGD, GOT and $\beta$-AMY isozymes. Running gels were $8.5 \%$ for 6-PGD and $\beta$-AMY, and $6 \%$ for GOT. A 5\% stacking gel was used for all three systems. The seed or leaf extracts were mixed with $0.005 \%$ bromo-phenol blue prior to electrophoresis, of which $30 \mu \mathrm{l}$ were utilized for electrophoresis using gel composition and running buffer protocols similar to those of Davis (1964). Electrophoresis was conducted on $1.5 \mathrm{~mm}$ thick gels, dimension $180 \times 160 \mathrm{~mm}$, on a Hoeffer vertical electrophoresis unit at $25 \mathrm{~mA} / \mathrm{gel}$ constant current with a temperature set at $4{ }^{\circ} \mathrm{C}$ until the blue dye front reached the end of the gel.

$S D S-P A G E$. High molecular weight glutenin sub-units were separated on $8.5 \%$ polyacrylamide gels in the presence of sodium dodecyl sulfate (SDS-PAGE) with seed extraction and gel composition details similar to Payne et al. (1980). Three percent stacking and 8.5\% running gels were used on a vertical Hoeffer electrophoresis unit with the same dimensions as for native-PAGE. The electrophoretic run was conducted at room temperature at $5 \mathrm{~mA} / \mathrm{gel}$ for $2 \mathrm{hr}$ followed by $10 \mathrm{~mA} / \mathrm{gel}$, until the dye front reached the end of the gel. The gels were rinsed in 30\% ethanol for $30 \mathrm{~min}$ to remove SDS and stained overnight in a $0.1 \%$ Coomassie brilliant blue R-250+50\% methanol $+10 \%$ acetic acid solution. Gels were destained in a $40 \%$ methanol $+10 \%$ acetic acid solution and stored in 10\% acetic acid.

Enzyme staining. Staining for EST-5, GPI, $\alpha$-AMY and $\beta$-AMY was according to William and Mujeeb-Kazi (1992) with the two AMY procedures being similar. The GOT isozyme was stained in $50 \mathrm{ml} 0.01 \mathrm{M}$ Tris- $\mathrm{HCl}$ (pH 7.4) containing $100 \mathrm{mg}$ pyruvic acid, $50 \mathrm{mg}$ $\alpha$-keto-glutamic acid with a well mixed $5 \mathrm{mg}$ of pyridoxyl-5-phosphate $+75 \mathrm{mg}$ fast blue BB salt addition just prior to staining. The 6-PGD staining solution comprised of $50 \mathrm{ml}$ Tris- $\mathrm{HCl}$ (pH 7.4) containing $10 \mathrm{mg}$ 6-phosphogluconic acid, $0.5 \mathrm{mg}$ NADP, $10 \mathrm{mg}$ MTT and $1 \mathrm{mg}$ PMS.

\section{Results and discussion}

High molecular weight glutenins (HMW-Glu). Identification and characterization of different allelic forms of high molecular weight glutenin subunits in bread wheat is important because of its implications on bread making quality. These forms are well characterized (Payne and Lawrence 1983). Ae. variabilis possessed two bands in the HMW-Glu region of lower mobility than the most common slow moving HMW-Glu subunits of bread wheat (Fig. 1). These two lower mobility bands were also expressed in the wheat/Ae. variabilis amphiploids. The HMW-Glu genes are located in the long arms of homoeologous group 1 wheat chromosomes (Beitz et al. 1975, Lawrence and Shepherd 1981). The two bands observed could therefore serve as markers for characterizing Ae. variabilis chromosomes homoeologous to 


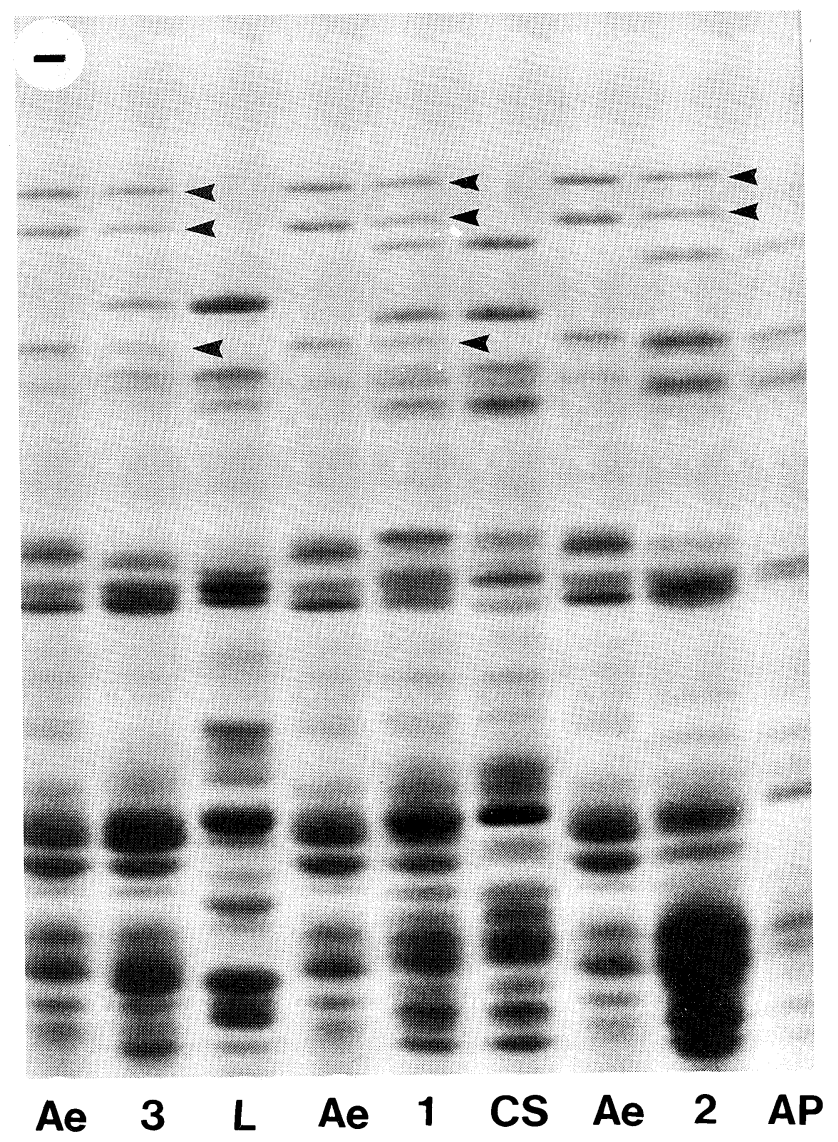

Fig. 1. Separation of high molecular weight glutenins of SDS-PAGE (8.5\%) gels of Triticum aestivum cv. Chinese Spring (CS), cv. Alondra/Pavon (AP), T. turgidum cv. Laru (L), Ae. variabilis (Ae) and the amphiploids of $\mathrm{CS} /$ Ae variabilis (1), $\mathrm{AP} /$ Ae. variabilis (2) and $\mathrm{L} / \mathrm{Ae}$. variabilis (3). Diagnostic marker bands are indicated by arrowheads.

group 1 of both bread and durum wheat.

Glucose phosphate isomerase (GPI). Glucose phosphate isomerase separation on isoelectric focusing gives 8 to 10 bands in bread wheat and 4 to 5 bands in durum cultivars (Chojecki and Gale 1982). Bread wheat cultivars have a distinctly different banding pattern than Ae. variabilis (Fig. 2). Both bread and durum wheat amphiploids with Ae. variabilis coexpress the wheat and Ae. variabilis bands. This marker can thus be utilized to characterize Ae. variabilis chromosomes and establish homoeologous relationships with wheat, since GPI genes are reported to locate on the homoeologous group 1 chromosomes of wheat (Hart 1979, Chojecki and Gale 1982).

Grain esterase (EST-5). Ae. variabilis expressed a multitude of bands for grain esterase (EST-5) of which several were unique for the species (Fig. 3). Two of these bands express in the basic region of the IEF gels and can potentially serve as diagnostic markers, since neither durum or bread wheats possess them. EST-5 genes are located in the long arms of homoeologous group 3 chromosomes of wheat (Ainsworth et al. 1984). Since the two marker bands (Fig. 3) were also expressed in the wheat/Ae. variabilis amphiploids, homoeology diagnosis of Ae. variabilis chromosomes with group 3 chromosomes of wheat is established.

6-Phosphogluconate dehydrogenase (6-PGD). Bread and durum wheat cultivars express a single 6-PGD band on native-PAGE (Fig. 4). Ae. variabilis possesses one overlapping band 


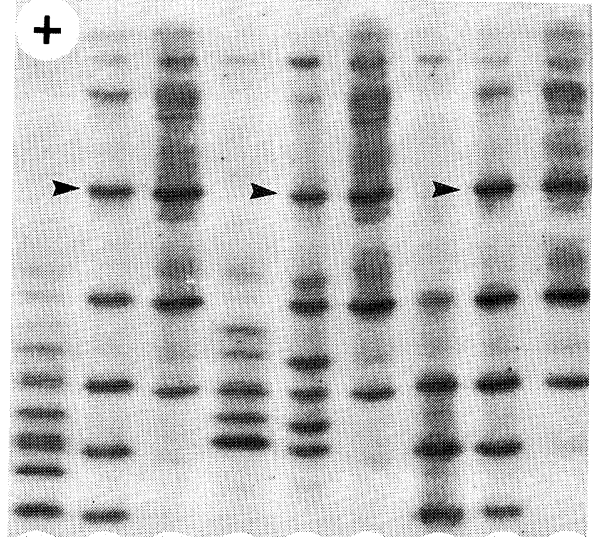

CS 1 Ae AP 2 Ae L 3 Ae

Fig. 2. Grain GPI banding profiles on isoelectric focusing gels of Triticum aestivum cv. Chinese Spring (CS), cv. Alondra/Pavon (AP), T. turgidum cv. Laru (L), Ae. variabilis (Ae) and the amphiploids of $\mathrm{CS} / A$ e. variabilis (1), $\mathrm{AP} /$ Ae. variabilis (2) and $\mathrm{L} /$ Ae . variabilis (3). Diagnostic marker bands are indicated by arrowheads.

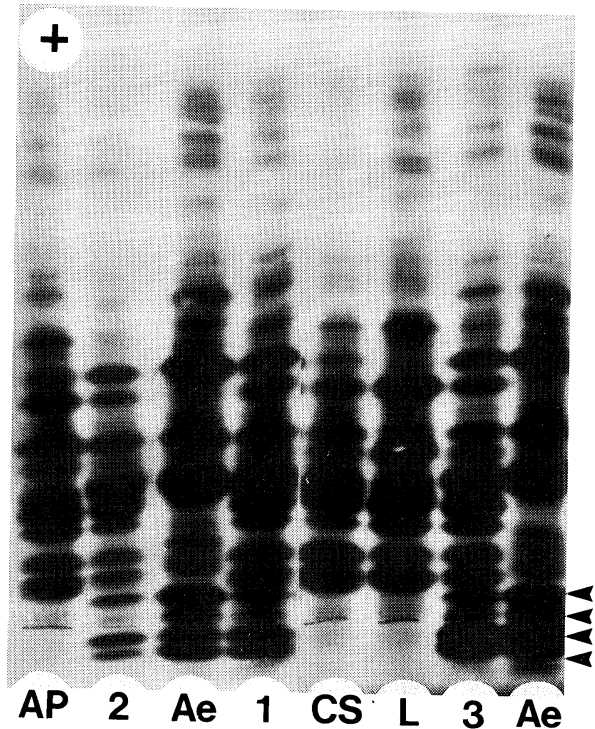

Fig. 3. Grain esterase (EST-5) banding profiles on IEF ( $\mathrm{pH} 3.5-9.5$ ) of Triticum aestivum cv. Chinese Spring (CS), cv. Alondra/Pavon (AP), T. turgidum cv. Laru (L), Ae. variabilis (Ae) and the amphiploids of $\mathrm{CS} /$ Ae. variabilis (1), AP/Ae. variabilis (2) and $\mathrm{L} / A$ e. variabilis (3). Diagnostic marker bands are indicated by arrowheads.

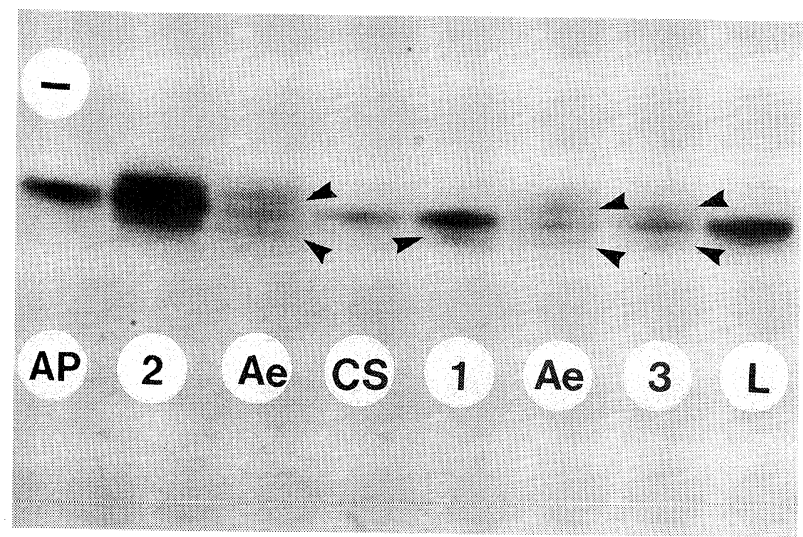

Fig. 4. Native-PAGE ( $8.5 \%$ acrylamide) of 6-phospho gluconate dehydrogenase isozymes of Triticum aestivum cv. Chinese Spring (CS), cv. Alondra/Pavon (AP), T. turgidum cv. Laru (L), Ae. variabilis (Ae) and the amphiploids of $\mathrm{CS} /$ Ae. variabilis (1), $\mathrm{AP} /$ Ae variabilis (2) and $\mathrm{L} /$ Ae. variabilis (3). Diagnostic marker bands are indicated by arrowheads.

with wheat and two distinctive bands; one each of slower or faster mobility than wheat. These bands were also present in the amphiploids and are anticipated to serve as identifiers of the presence of Ae. variabilis chromosomes with homoeology to group 6 or 7 of wheat. Genes for 6-PGD are located on chromosomes 6A, 6BL and 7BL in bread wheat (Hsam and Zeller 1982).

$\beta$-Amylase $(\beta-A M Y)$. The T. aestivum cultivars Chinese Spring and Alondra/Pavon expressed a multitude of bands when $\beta$-AMY was separated on IEF, pH 4.0-6.5 (Fig. 5). There were two to three diagnostic bands for Ae. variabilis, that distinguished it from the respective wheat cultivars. The wheat/Ae. variabilis amphiploids also expressed this $A$ e. 


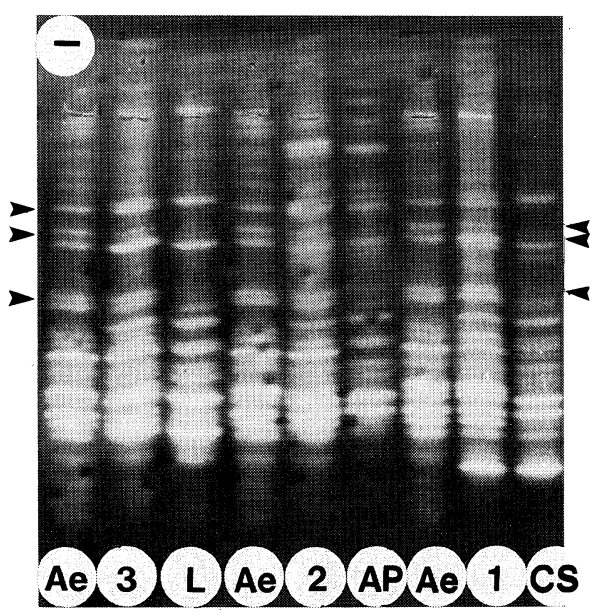

Fig. 5. Separation of $\beta$-AMY isozymes on IEF gels (pH 4.0-6.5) of Triticum aestivum cv. Chinese Spring (CS), cv. Alondra/Pavon (AP), T. turgidum cv. Laru (L), Ae. variabilis (Ae) and the amphiploids of CS/Ae. variabilis (1), AP/Ae. variabilis (2) and L/Ae. variabilis (3). Diagnostic marker bands are indicated by arrowheads.

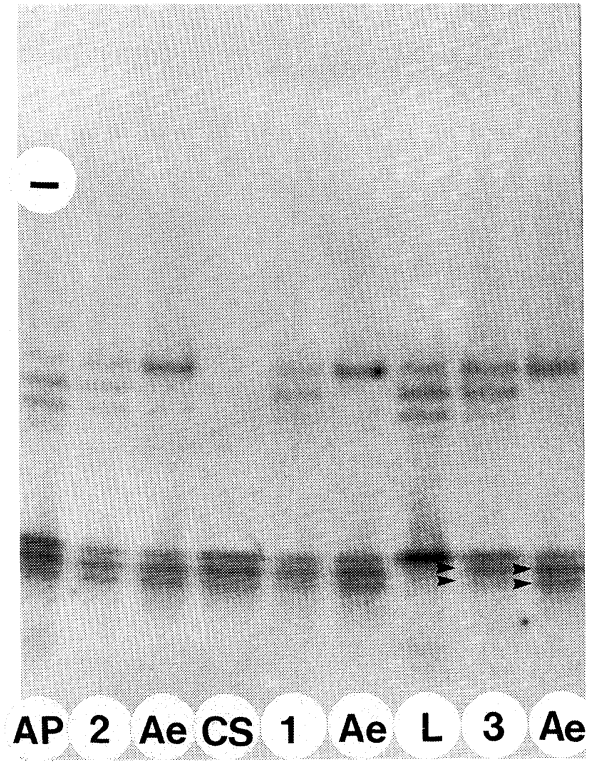

Fig. 6. Native-PAGE ( $8.5 \%$ acrylamide) glutamate oxaloacetate transaminase isozymes of Triticum aestivum cv. Chinese Spring (CS), cv. Alondra/Pavon (AP), T. turgidum cv. Laru (L), Ae. variabilis (Ae) and the amphiploids of $\mathrm{CS} /$ Ae. variabilis (1), $\mathrm{AP} / A e$. variabilis (2) and L/Ae. variabilis (3). Diagnostic marker bands are indicated by arrowheads.

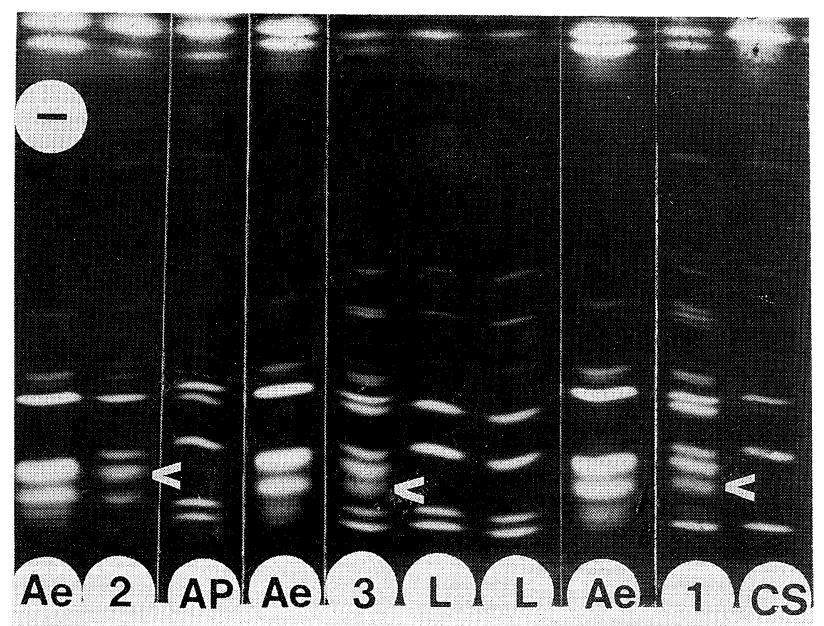

Fig. 7. IEF ( $\mathrm{pH}$ 4.0-6.5) separation of $\alpha$-AMY isozymes of Triticum aestivum cv. Chinese Spring (CS), Alondra/Pavon (AP), T. turgidum cv. Laru (L), Ae. variabilis (Ae) and the amphiploids of CS/Ae. variabilis (1), AP/Ae. variabilis (2) and L/Ae. variabilis (3). Diagnostic marker bands are indicated by arrowheads.

variabilis specific band (Fig. 5). $\beta$-AMY genes are located on wheat chromosomes 4A $\beta, 4 \mathrm{DL}$ ( $\beta$-Amy 1) and 5AL ( $\beta$-Amy 2), (Ainsworth et al. 1983). The above Ae. variabilis specific band marker provides us with the diagnostic tool for identifying Ae. variabilis chromosomes homoeologous to group 4 or 5 chromosomes of wheat. 
Glutamate oxaloacetate transaminase (GOT). When GOT isozymes are separated on native-PAGE, bread wheats exhibit three bands in the GOT-2 region (Hart 1975). GOT-2 isozyme genes are located in homoeologous group 6 chromosomes of wheat. These three bands arise by a recombination of monomeric subunits encoded by genes present in the different group 6 chromosomes to form an active dimeric GOT enzyme (Hart 1975). Ae. variabilis has 3 bands in the GOT-2 region of comparable staining intensities to the GOT-2 region of bread wheat (Fig. 6) and do not provide a positive marker. Durum wheats (T. turgidum) however, have a single band and the durum/Ae. variabilis amphiploid possess three bands in the GOT-2 region that can be potential markers to diagnose the presence of Ae. variabilis chromosomes homoeologous to group 6 of wheat.

$\alpha$-Amylase $(\alpha-A M Y)$. There are two zones of activity when $\alpha$-amylase isozymes are separated on IEF (Nishikawa and Nobuhara 1971, Gale et al. 1983). The banding patterns of $\alpha$-AMY 2 (low pI region) showed differences between wheat and Ae. variabilis (Fig. 7) that further expressed in their amphiploids. $\alpha$-AMY genes are located in homoeologous group 6 ( $\alpha$-Amy 1 locus) and group 7 chromosomes ( $\alpha$-Amy 2 locus) of wheat (Nishikawa and Nobuhara 1971, Gale et al. 1983). The $\alpha$-AMY 2 isozyme differences present can thus be used to characterize Ae. variabilis chromosomes with homoeology to group 7 chromosomes of wheat.

\section{Summary}

Aegilops variabilis $(2 \mathrm{n}=4 \mathrm{x}=28$, UUSS) with a high level of resistance to Karnal bunt (Tilletia indica); an important soil-borne disease; is a potential genetic variability source for transfer to wheat (Triticum aestivum L.). Diagnostic markers using proteins and isozymes have hence been established to detect the presence of Ae. variabilis chromosomes in Triticum background. High molecular weight glutenins (HMW-Glu), glucose phosphate isomerase (GPI), seed esterase (EST-5), glutamate oxaloacetate transaminase (GOT), 6-phosphogluconate dehydrogenase (6-PGD), $\alpha$-amylase ( $\alpha$-AMY) and $\beta$-amylase ( $\beta$-AMY) were positive biochemical markers. These markers enable identification of the disomic chromosome additions of Ae. variabilis to wheat and also determine their homoeology with the homoeologous groups of wheat following evaluation of their respective amphiploids; T. aestivum/Ae. variabilis $(2 \mathrm{n}=10 \mathrm{x}=70)$ and $T$. turgidum/Ae. variabilis $(2 \mathrm{n}=8 \mathrm{x}=56)$.

Key words: Alien genetic variation, Biochemical markers, Intergeneric hybridization, Triticeae, Wheat improvement.

\section{References}

Ainsworth, C. C., Gale, M. D. and Baird, S. 1983. The genetics of $\beta$-amylase isozymes in wheat. Theor. Appl. Genet. 66: 39-49.

- , - and - 1984. The genetic control of grain esterase in hexaploid wheat. Theor. Appl. Genet. 68: 219-226.

Beitz, J. A., Shepherd, K. W. and Wall, J. S. 1975. Single-kernel analysis of glutenin: Use in wheat genetics and breeding. Cereal Chem. 52: 513-532.

Chojecki, A. J. S. and Gale, M. D. 1982. Genetic control of glucose phosphate isomerase in wheat and related species. Heredity 49: 337-347.

Davis, B. J. 1964. Disc electrophoresis. II. Methods and application to human serum proteins. Ann. Acad. Sci. 121: 404-427.

Gale, M. D., Law, C. N., Chojecki, A. J. and Kempton, R. A. 1983. Genetic control of $\alpha$-amylase production in wheat. Theor. Appl. Genet. 64: 309-316.

Hart, G. E. 1975. Glutamate oxaloacetate transaminase isozymes in Triticum: Evidence for multiple systems of triplicate structural genes in hexaploid wheat. In: Isozymes III Developmental Biology. C. Market (ed.) Acad. Press Inc., New York. pp. 637-657. 
- 1979. Evidence for a triplicate set of glucose phosphate isomerase structural genes in hexaploid wheat. Biochem. Genet. 17: 585-598.

Hsam, S. L. K. and Zeller, F. J. 1982. Genetic control of 6-phosphogluconate dehydrogenase (6-PGD) isozymes in cultivated wheat and rye. Theor. Appl. Genet. 62: 317-320.

Lawrence, G. J. and Shepherd, K. W. 1981. Chromosomal location of genes controlling seed proteins in species related to wheat. Theor. Appl. Genet. 59: 25-31.

Mujeeb-Kazi, A. and Asiedu, R., 1990. Wide hybridization-potential of alien genetic transfers for Triticum aestivum improvement. In: Biotechnology in Agriculture and Forestry, vol. 13. Y. P. S. Bajaj (ed.) Springer, Berlin, pp. 111-127.

Nishikawa, K. and Nobuhara, M. 1971. Genetic studies of $\alpha$-amylase isozymes in wheat. 1. location of genes and variation in tetra- and hexaploid wheat. Jpn. J. Genet. 46: 345-353.

Payne, P. I. and Lawrence, C. J. 1983. Catalogue of alleles for the complex gene loci, Glu-A-1, Glu-B-1 and Glu-D-1 which code for the high-molecular-weight subunits of glutenin in hexaploid wheat. Cereal Res. Commun. 11 29-35.

-, Law, C. N. and Mudd, E. E. 1980. Control by homoeologous group 1 chromosomes of the high-molecular-weight subunits of glutenin, a major protein of wheat endosperm. Theor. Appl. Genet. 58: 113-120.

Warham, E. J., Mujeeb-Kazi, A. and Rosas, V. 1986. Karnal bunt (Tilletia indica) resistance screening of Aegilops species and their practical utilization for Triticum aestivum improvement. Can. J. Plant Pathol. 8: 65-70.

William, M. D. H. M. and Mujeeb-Kazi, A. 1992. Isozyme and cytological markers of some Psathyrostachys juncea accessions. Theor. Appl. Genet. 84: 528-534.

- and - 1993. Thinopyrum bessarabicum: Biochemical and cytological markers for the detection of genetic introgression in its hybrid derivatives with Triticum aestivum L. Theor. Appl. Genet. 86: 365-370. 